\title{
Superconvergence of FEM for Distributed Order Time Fractional Variable Coefficient Diffusion Equations
}

\author{
Yanhua Yang and Jincheng Ren*
}

\begin{abstract}
In this paper, a numerical fully discrete scheme based on the finite element approximation for the distributed order time fractional variable coefficient diffusion equations is developed and a complete error analysis is provided. The weighted and shifted Grünwald formula is applied for the time-fractional derivative and finite element approach for the spatial discretization. The unconditional stability and the global superconvergence estimate of the fully discrete scheme are proved rigorously. Extensive numerical experiments are presented to illustrate the accuracy and efficiency of the scheme, and to verify the convergence theory.
\end{abstract}

\section{Introduction}

Recently, there has been growing interest in fractional differential equations (FDEs) because their widespread applications in describing processes arising from various fields of physics, finance, material science, control theory, viscoelasticity, chaos and others are filling up quickly, see, e.g., $9,12,13,25,30,32$. Analytical methods, such as the Fourier transform method and the Laplace transform method, have been used to obtain closedform solutions to FDEs [20,24]. However, analytical methods do not work well on the majority of FDEs. Therefore, it is of great importance to find efficient and reliable numerical methods to solve these FDEs.

It is pointed out recently that the time-fractional anomalous diffusion equation with a constant-order temporal derivative cannot describe processes lacking temporal scaling 3 , 4]. In other words, the single-order time-fractional diffusion equation cannot characterize this process and it must be generalized. The distributed-order time-fractional diffusion equation turns out to be a good alternative choice. The distributed-order differential equation can also be regarded as a natural generalization of the single-order and the

Received December 7, 2017; Accepted June 19, 2018.

Communicated by Suh-Yuh Yang.

2010 Mathematics Subject Classification. 65N15, 65N30.

Key words and phrases. distributed order diffusion equations, finite element method, fully discrete scheme, superconvergence estimate.

The research is supported by National Natural Science Foundation of China (No. 11601119), sponsored by Program for HASTIT (No. 18HASTIT027) and Young talents Fund of HUEL.

${ }^{*}$ Corresponding author. 
multi-term FDEs [7]. Many numerical approaches to the single-order and the multi-term FDEs have been proposed in the literature. Zhuang et al. [35] introduced a new way for solving sub-diffusion equation by integration of the original equation on the both sides to obtain an implicit numerical method. The stability and convergence of the scheme were proved by the energy method. In [34], Zhang et al. constructed a Crank-Nicolson-type difference scheme and a compact difference scheme for solving the time fractional subdiffusion equation with Riemann-Liouville fractional derivative, respectively. They proved that the two difference schemes were unconditionally stable and the numerical solution was convergent in the maximum norm. Cui [6] and Zhang et al. 33] constructed alternating direction implicit scheme and compact alternating direction implicit scheme for solving the two-dimensional time fractional sub-diffusion equation, respectively. Inspired by [18] on the weighted and shifted Grünwald difference operator, Wang and Vong [27,28] established high order schemes for the fractional diffusion-wave equation and the modified anomalous fractional subdiffusion equation and fractional Klein-Gordon equation, respectively. For the multi-term time-fractional diffusion equation, Liu et al. [17 firstly reformulated the original multi-term problem into a system of single-term fractional differential equations and a fractional predictor-corrector method was then used to solve the system. Recently, Ren and Sun $[22,23$ provided the high-order spatially compact scheme for multi-term time-fractional sub-diffusion equations and diffusion-wave equation, respectively.

However, the discussion on the numerical methods for the time-fractional diffusion equation of distributed order is meager. Now we review some recent contributions on this issue. Liao et al. 15 derived a Du Fort-Frankel type explicit scheme for solving the distributed order subdiffusion equation by combining the L1 formula of RiemannLiouville derivative with the midpoint quadrature of the weighted integral. Based on the L1 approximation for the Caputo fractional derivative in temporal direction and fractional centered difference discretization Riesz derivative, Liu et al. 29 investigated the timespace distributed order fractional diffusion equations. Using the weighted and shifted Grünwald formula proposed in [26], some effective difference schemes are developed for onedimensional and two-dimensional distributed order FPDEs. The unconditional stability and convergence of the obtained schemes are investigated using the energy method in $10,11,14$.

The usual way of analyzing superconvergence properties of postprocessed computed solutions consists of two steps: (1) Supercloseness property: an interpolation approximating the finite element solution of higher order. Often such an interpolation does exist if the underlying mesh has a special structure. (2) A postprocessing operator: an interpolation operator (in a higher order finite element space) with certain stability, invariance and higher order approximation properties. Applying this interpolation operator to the 
original finite element solution, we obtain the postprocessed solution, which has a superconvergence property (cf. 8]). Although the superconvergence property was widely discussed in classical PDEs and achieved fruitful results, to our knowledge, the superclose and global superconvergence properties have not been proved for finite element schemes of distributed order FPDEs. This motivates us to consider the superclose and global superconvergence properties of this equations. The main purpose of this paper is to obtain the superconvergence of fully discrete finite element approximation for the FPDEs of distributed order. The weighted and shifted Grünwald formula (cf. [26, 27]) is applied for the time-fractional derivative and the finite element approach works for the spatial discretization. Based on the integral identity technique developed in [16], the global superconvergence error estimates for variable coefficient fractional subdiffusion equations are proved rigorously.

The plan of this paper is as follows. In Section 2 , some preliminary numerical quadrature formulae and useful lemmas are prepared. In Section 3, a fully discrete scheme for the distributed order fractional equations is developed. The unconditional stability and superconvergence of the obtained scheme are proved in Section 4. In Section 5, some numerical examples are presented to verify our theoretical results. Some conclusions are given in the last section. Throughout, the notation $c$ denotes a generic constant, which may not be the same at different occurrences, but it is always independent of the mesh size $h$, the time step size $\tau$ and $\Delta \alpha$.

\section{Preliminaries}

In this section, some useful notations, lemmas and formulae will be prepared for the forthcoming work.

We denote by $W^{k, p}(\Omega)$ the standard Sobolev space of $k$-differential functions in $L^{p}(\Omega)$, its norm by $\|\cdot\|_{k, p}$, and the norm of $H^{k}(\Omega)$ by $\|\cdot\|_{k}$. When $k=0$, we let $L^{2}(\Omega)$ denote the corresponding space defined on $\Omega$ with norm $\|\cdot\|$. Let $C_{0}^{\infty}(\Omega)$ stand for the space of smooth functions with compact support in $\Omega$, and $H_{0}^{s}(\Omega)$ denote the closure of $C_{0}^{\infty}(\Omega)$ with respect to norm $\|\cdot\|_{s}$ (cf. [1] for details).

For the finite difference discretization of the distributed order fractional derivative, we divide the interval $[0,1]$ into $2 J$-subintervals with $\Delta \alpha=1 /(2 J)$ and $\alpha_{l}=l \Delta \alpha, l=$ $0,1,2, \ldots, 2 J$. Similarly, the interval $[0, T]$ is divided into $N$-subintervals with $\tau=T / N$ and $t_{k}=k \tau, k=0,1,2, \ldots, N$. Let $u^{n}$ denote the solution $u(x, y, t)$ at $t=t_{n}$.

To describe the numerical approximation, several formulae and lemmas are presented below. 
Lemma 2.1 (The composite trapezoid formula). Let $s(\alpha) \in C^{2}[0,1]$, then we have

$$
\int_{0}^{1} s(\alpha) d \alpha=\Delta \alpha \sum_{l=0}^{2 l} c_{l} s\left(\alpha_{l}\right)-\frac{(\Delta \alpha)^{2}}{12} s^{\prime \prime}(\xi), \quad \xi \in(0,1),
$$

where $c_{0}=c_{2 J}=1 / 2, c_{l}=1, l=1,2, \ldots, 2 J-1$.

Denote

$$
\mathscr{C}^{n+\alpha}(\mathbb{R})=\left\{f \mid f \in L^{1}(\mathbb{R}) \text { and } \int_{-\infty}^{\infty}(1+|\kappa|)^{n+\alpha}|\widehat{f}(\kappa)| d \kappa<\infty\right\},
$$

where $\widehat{f}(\kappa)=\int_{-\infty}^{\infty} e^{i \kappa t} f(t) d t$ is the Fourier transformation of $f(t)$.

Lemma 2.2 (The Grünwald formula). [19] Suppose that $f \in \mathscr{C}^{1+\alpha}(\mathbb{R})$, and let

$$
{ }_{-\infty} D_{t}^{\alpha} f(t)=\frac{1}{\Gamma(1-\alpha)} \frac{d}{d t} \int_{-\infty}^{t} \frac{f(s)}{(t-s)^{\alpha}} d s
$$

and

$$
A_{\tau, r}^{\alpha} f(t)=\frac{1}{\tau^{\alpha}} \sum_{k=0}^{\infty} g_{k}^{(\alpha)} f(t-(k-r) \tau)
$$

where $r$ is an integer and $g_{k}^{(\alpha)}=(-1)^{k}\left(\begin{array}{l}\alpha \\ k\end{array}\right)$. Then

$$
A_{\tau, r}^{\alpha} f(t)={ }_{-\infty} D_{t}^{\alpha} f(t)+O(\tau)
$$

uniformly holds in $t \in \mathbb{R}$ as $\tau \rightarrow 0$.

Lemma 2.3. 26, 27 Suppose that $f \in \mathscr{C}^{2+\alpha}(\mathbb{R})$, then we get

$$
\left(1+\frac{\alpha}{2}\right) A_{\tau, 0}^{\alpha} f(t)-\frac{\alpha}{2} A_{\tau,-1}^{\alpha} f(t)=\frac{1}{\tau^{\alpha}} \sum_{k=0}^{\infty} \lambda_{k}^{(\alpha)} f(t-k \tau)={ }_{-\infty} D_{t}^{\alpha} f(t)+O\left(\tau^{2}\right)
$$

uniformly holds in $t \in \mathbb{R}$ as $\tau \rightarrow 0$, where

$$
\lambda_{0}^{(\alpha)}=\left(1+\frac{\alpha}{2}\right) g_{0}^{(\alpha)}, \quad \lambda_{k}^{(\alpha)}=\left(1+\frac{\alpha}{2}\right) g_{k}^{(\alpha)}-\frac{\alpha}{2} g_{k-1}^{(\alpha)}, \quad k \geq 1 .
$$

We then have the following property on $\lambda_{k}^{(\alpha)}$.

Lemma 2.4. 11,27 Let $\lambda_{k}^{(\alpha)}$ be defined by 2.1), then for any positive integer $k$ and real vector $\left(v_{0}, v_{1}, v_{2}, \ldots, v_{m}\right)^{T} \in \mathbb{R}^{m+1}$, it holds that

$$
\sum_{n=0}^{m}\left(\sum_{k=0}^{n} \lambda_{k}^{(\alpha)} v_{n-k}\right) v_{n} \geq 0
$$

Lemma 2.5. 11 Let $\mu=\Delta \alpha \sum_{l=0}^{2 J} c_{l} w\left(\alpha_{l}\right) \cdot \frac{1}{\tau^{\alpha} l} \lambda_{0}^{\left(\alpha_{l}\right)}$, it holds that

$$
\mu=\frac{1}{O(\tau|\ln \tau|)} \text {. }
$$




\section{Construction the fully discrete finite element scheme}

This section is devoted to obtain the stability analysis of the time distributed order fractional diffusion equations.

Consider the following fractional diffusion equations

$$
\begin{cases}\mathcal{D}_{t}^{[w]} u(x, y, t)=\nabla \cdot(b(x, y) \nabla u(x, y, t))+g(x, y, t) & (x, y) \in \Omega, t \in(0, T], \\ u(x, y, 0)=0 & (x, y) \in \Omega, \\ \left.u(x, y, t)\right|_{\partial \Omega}=0 & t \in[0, T],\end{cases}
$$

where $\Omega \subset \mathbb{R}^{2}$ is a rectangular domain with boundary $\partial \Omega$ parallel to the $x$-axis or $y$-axis in the plane and $(0, T]$ is the time interval. The symbols $\nabla \cdot$ and $\nabla$ denote the divergence and gradient operators, respectively. The function $b(x, y)$ is assumed to be smooth, bounded and satisfy following assumption

$$
0<\beta_{1} \leq b(x, y) \leq \beta_{2}
$$

Here, $\mathcal{D}_{t}^{[w]} u$ denotes the distributed order fractional derivative of $u$ in time $t$ (with respect to the weight function $w$ ) defined by

$$
\begin{aligned}
\mathcal{D}_{t}^{[w]} u(x, y, t) & =\int_{0}^{1} w(\alpha){ }_{0}^{C} D_{t}^{\alpha} u(x, y, t) d \alpha, \\
w(\alpha) \geq 0, & \int_{0}^{1} w(\alpha) d \alpha=\omega_{0}>0, \\
{ }_{0}^{C} D_{t}^{\alpha} u(x, y, t) & = \begin{cases}\frac{1}{\Gamma(1-\alpha)} \int_{0}^{t}(t-s)^{-\alpha} \frac{\partial u(x, y, s)}{\partial s} d s & 0 \leq \alpha<1, \\
\frac{\partial u(x, y, t)}{\partial t} & \alpha=1 .\end{cases}
\end{aligned}
$$

It is without loss of generality to assume that $u(x, y, 0)=0$. If $u(x, y, 0)=\phi(x, y) \neq 0$, we can let $v(x, y, t)=u(x, y, t)-\phi(x, y)$ and consider the problem with respect to $v(x, y, t)$.

Based on the method introduced in [11], we describe a discrete scheme for problem (3.1). Suppose $w(\alpha) \in C^{2}[0,1]$ and ${ }_{0}^{C} D_{t}^{\alpha} u \in C^{2}\left([0, T] ; H^{m+2}(\Omega) \cap H_{0}^{1}(\Omega)\right)$. By Lemma 2.1. we have

$$
\begin{aligned}
\mathcal{D}_{t}^{[w]} u\left(x, y, t_{k}\right)= & \int_{0}^{1} w(\alpha){ }_{0}^{C} D_{t}^{\alpha} u\left(x, y, t_{k}\right) d \alpha \\
= & \Delta \alpha \sum_{l=0}^{2 J} c_{l} w\left(\alpha_{l}\right){ }_{0}^{C} D_{t}^{\alpha_{l}} u\left(x, y, t_{k}\right) \\
& -\left.\frac{(\Delta \alpha)^{2}}{12} \frac{\partial^{2}\left[w(\alpha){ }_{0}^{C} D_{t}^{\alpha} u\left(x, y, t_{k}\right)\right]}{\partial \alpha^{2}}\right|_{\alpha=\xi^{(k)}} \\
= & \Delta \alpha \sum_{l=0}^{2 J} c_{l} w\left(\alpha_{l}\right){ }_{0}^{C} D_{t}^{\alpha_{l}} u\left(x, y, t_{k}\right)+O\left((\Delta \alpha)^{2}\right),
\end{aligned}
$$


where $\xi^{(k)} \in(0,1)$.

In the current work, we assume that the solution $u(x, y, t)$ can be extended by zero from the bounded time domain $[0, T]$ to $\mathbb{R}$. Noticing the equivalence between the RiemannLiouville fractional derivative ${ }_{-\infty} D_{t}^{\alpha} f(t)$ with $f(t)=0$ when $t \leq 0$ and the Caputo fractional derivative ${ }_{0}^{C} D_{t}^{\alpha} f(t)$, by Lemma 2.3 and (3.4), we obtain

$$
\mathcal{D}_{t}^{[w]} u\left(x, y, t_{k}\right)=\Delta \alpha \sum_{l=0}^{2 J} c_{l} w\left(\alpha_{l}\right)\left[\frac{1}{\tau^{\alpha_{l}}} \sum_{j=0}^{k} \lambda_{j}^{\left(\alpha_{l}\right)} u\left(x, y, t_{k-j}\right)+O\left(\tau^{2}\right)\right]+O\left((\Delta \alpha)^{2}\right) .
$$

Before establishing fully discrete finite element scheme for the problem (3.1), we first introduce some more notations as follows.

Let $\Im_{h}$ be a family of rectangular subdivisions such that $\bar{\Omega}=\bigcup_{K \in \Im_{h}} \bar{K}$. We use $h$ to denote the maximum diameter of the rectangular subdivisions. Let $V_{h} \subset H_{0}^{1}(\Omega)$ be the standard finite element space consisting of continuous, piecewise polynomial of degree $m$, that vanish on the entire boundary $\partial \Omega$. Moreover, denoting $I_{h}: C(\bar{\Omega}) \rightarrow V_{h}$ the Lagrange interpolation operator, the interpolation error estimates imply that for $u \in H^{r+1}(\Omega)$ (cf. 5)

$$
\left\|u-I_{h} u\right\|_{s} \leq c h^{r-s}\|u\|_{r+1}, \quad 0 \leq s \leq r, 0<r \leq m .
$$

Then the fully discrete finite element for the problem (3.1) can be formulated as: find $U_{h}^{k} \in V_{h}$ such that

$$
\begin{aligned}
\Delta \alpha \sum_{l=0}^{2 J} c_{l} w\left(\alpha_{l}\right) \frac{1}{\tau^{\alpha_{l}}} \sum_{j=0}^{k} \lambda_{j}^{\left(\alpha_{l}\right)}\left(U_{h}^{k-j}, v_{h}\right)+\left(b \nabla U_{h}^{k}, \nabla v_{h}\right) & =\left(g^{k}, v_{h}\right), \quad \forall v_{h} \in V_{h}, \\
U_{h}^{0} & =0 .
\end{aligned}
$$

Next, we focus on the stability analysis of the fully discrete scheme (3.6). In this regard, we have the following theorem.

Theorem 3.1. The fully discrete finite element scheme $(3.6)$ is unconditionally stable with respect to the inhomogeneous term $g$. That is to say

$$
\tau \sum_{k=1}^{n}\left\|\nabla U_{h}^{k}\right\|^{2} \leq c \tau \sum_{k=1}^{n}\left\|g^{k}\right\|^{2}, \quad \forall 1 \leq n \leq N .
$$

Proof. Taking $v_{h}=U_{h}^{k}$ in 3.6 , using Cauchy-Schwarz inequality and noticing the equivalence of norms in $H_{0}^{1}(\Omega)$, we have

$$
\begin{aligned}
& \Delta \alpha \sum_{l=0}^{2 J} c_{l} w\left(\alpha_{l}\right) \frac{1}{\tau^{\alpha_{l}}} \sum_{j=0}^{k} \lambda_{j}^{\left(\alpha_{l}\right)}\left(U_{h}^{k-j}, U_{h}^{k}\right)+\left(b \nabla U_{h}^{k}, \nabla U_{h}^{k}\right) \\
= & \left(g^{k}, U_{h}^{k}\right) \\
\leq & c\left\|g^{k}\right\|^{2}+\frac{\beta_{1}}{2}\left\|\nabla U_{h}^{k}\right\|^{2}, \quad \forall 1 \leq k \leq N .
\end{aligned}
$$


For the second term on the left-hand side of (3.7), noticing $(3.2)$, we have

$$
\left|\left(b \nabla U_{h}^{k}, \nabla U_{h}^{k}\right)\right| \geq \beta_{1}\left\|\nabla U_{h}^{k}\right\|^{2} .
$$

Summing up the above inequality for $k$ from 1 to $n$, we obtain

$$
\begin{aligned}
& \Delta \alpha \sum_{l=0}^{2 J} c_{l} w\left(\alpha_{l}\right) \frac{1}{\tau^{\alpha_{l}}} \sum_{k=1}^{n} \sum_{j=0}^{k} \lambda_{j}^{\left(\alpha_{l}\right)}\left(U_{h}^{k-j}, U_{h}^{k}\right)+\frac{\beta_{1}}{2} \sum_{k=1}^{n}\left\|\nabla U_{h}^{k}\right\|^{2} \\
\leq & c \sum_{k=1}^{n}\left\|g^{k}\right\|^{2}, \quad \forall 1 \leq n \leq N .
\end{aligned}
$$

Adding $\mu\left(U_{h}^{0}, U_{h}^{0}\right)$ on both sides of the above inequality, we get

$$
\begin{aligned}
& \Delta \alpha \sum_{l=0}^{2 J} c_{l} w\left(\alpha_{l}\right) \frac{1}{\tau^{\alpha_{l}}} \sum_{k=0}^{n} \sum_{j=0}^{k} \lambda_{j}^{\left(\alpha_{l}\right)}\left(U_{h}^{k-j}, U_{h}^{k}\right)+\frac{\beta_{1}}{2} \sum_{k=1}^{n}\left\|\nabla U_{h}^{k}\right\|^{2} \\
\leq & \mu\left(U_{h}^{0}, U_{h}^{0}\right)+c \sum_{k=1}^{n}\left\|g^{k}\right\|^{2}, \quad \forall 1 \leq n \leq N .
\end{aligned}
$$

When $\tau$ is sufficiently small, using Lemmas 2.4 and 2.5 yield

$$
\tau \sum_{k=1}^{n}\left\|\nabla U_{h}^{k}\right\|^{2} \leq \frac{2 \mu \tau}{\beta_{1}}\left\|U_{h}^{0}\right\|^{2}+c \tau \sum_{k=1}^{n}\left\|g^{k}\right\|^{2}, \quad \forall 1 \leq n \leq N .
$$

Noticing $U_{h}^{0}=0$, the desired result is obtained. This completes the proof.

4. Superconvergence estimate for the fully discrete finite element scheme

In this section, we state an error estimate for the fully discrete scheme. In order to analyze the spatial discretization error, we assume that the solution is sufficiently smooth.

To begin, we introduce two lemmas, which play a very important role in proving supercloseness analysis.

Lemma 4.1. 16 Assume that $u \in H^{m+2}(\Omega)$, then we have

$$
\left|\left(\nabla\left(u-I_{h} u\right), \nabla v\right)\right| \leq c h^{m+1}\|u\|_{m+2}\|\nabla v\|, \quad \forall v \in V_{h} .
$$

Lemma 4.2. Suppose that ${ }_{0}^{C} D_{t}^{\alpha} u \in C^{2}\left([0, T] ; H^{m+1}(\Omega) \cap H_{0}^{1}(\Omega)\right), I_{h} u$ is the finite element interpolation of function $u$. Then we have for $1 \leq k \leq N$

$$
\begin{aligned}
& \left|\left(\mathcal{D}_{t}^{[w]} u^{k}-\Delta \alpha \sum_{l=0}^{2 J} c_{l} w\left(\alpha_{l}\right) \frac{1}{\tau^{\alpha_{l}}} \sum_{j=0}^{k} \lambda_{j}^{\left(\alpha_{l}\right)} I_{h} u^{k-j}, v\right)\right|^{2} \\
& \leq c\left(\tau^{2}+(\Delta \alpha)^{2}+h^{m+1}\right)^{2}\left(1+\max _{\substack{0 \leq s \leq T \\
0 \leq \alpha \leq 1}}\left\|_{0}^{C} \mathcal{D}_{t}^{\alpha} u(s)\right\|_{m+1}\right)^{2}+\frac{\beta_{1}}{4}\|\nabla v\|^{2}, \quad \forall v \in V_{h} .
\end{aligned}
$$


Proof. Using the triangle inequality, we have the following equation

$$
\begin{aligned}
& \left|\left(\mathcal{D}_{t}^{[w]} u^{k}-\Delta \alpha \sum_{l=0}^{2 J} c_{l} w\left(\alpha_{l}\right) \frac{1}{\tau^{\alpha_{l}}} \sum_{j=0}^{k} \lambda_{j}^{\left(\alpha_{l}\right)} I_{h} u^{k-j}, v\right)\right| \\
\leq & \left|\left(\mathcal{D}_{t}^{[w]} u^{k}-\Delta \alpha \sum_{l=0}^{2 J} c_{l} w\left(\alpha_{l}\right) \frac{1}{\tau^{\alpha_{l}}} \sum_{j=0}^{k} \lambda_{j}^{\left(\alpha_{l}\right)} u^{k-j}, v\right)\right| \\
& +\left|\Delta \alpha \sum_{l=0}^{2 J} c_{l} w\left(\alpha_{l}\right) \frac{1}{\tau^{\alpha_{l}}} \sum_{j=0}^{k} \lambda_{j}^{\left(\alpha_{l}\right)}\left(\left(I-I_{h}\right) u^{k-j}, v\right)\right| \\
\equiv & \left|\left(R_{1}^{k}, v\right)\right|+\left|\left(R_{2}^{k}, v\right)\right|, \quad \forall 1 \leq k \leq N .
\end{aligned}
$$

By (3.4) and the Cauchy-Schwarz inequality, noticing the equivalence of norms in $H_{0}^{1}(\Omega)$, we obtain

$$
\begin{aligned}
\left|\left(R_{1}^{k}, v\right)\right| & \leq c\left(\tau^{2}+(\Delta \alpha)^{2}\right)\|v\| \\
& \leq c\left(\tau^{2}+(\Delta \alpha)^{2}\right)^{2}+\frac{\beta_{1}}{8}\|\nabla v\|^{2}, \quad \forall 1 \leq k \leq N .
\end{aligned}
$$

It follows from (3.3) and Lemma 2.1 that

$$
\begin{aligned}
\left|\Delta \alpha \sum_{l=0}^{2 J} c_{l} w\left(\alpha_{l}\right)\right| & \leq\left|\Delta \alpha \sum_{l=0}^{2 J} c_{l} w\left(\alpha_{l}\right)-\int_{0}^{1} w(\alpha) d \alpha\right|+\int_{0}^{1} w(\alpha) d \alpha \\
& \leq O\left((\Delta \alpha)^{2}\right)+\omega_{0} \\
& \leq 1+\omega_{0} .
\end{aligned}
$$

In view of Lemma 2.3 , when $\tau$ is sufficiently small, we note that $1 \leq k \leq N$

$$
\begin{aligned}
\left|\frac{1}{\tau^{\alpha_{l}}} \sum_{j=0}^{k} \lambda_{j}^{\left(\alpha_{l}\right)} u^{k-j}\right| & \leq\left|\frac{1}{\tau^{\alpha_{l}}} \sum_{j=0}^{k} \lambda_{j}^{\left(\alpha_{l}\right)} u^{k-j}-{ }_{0}^{C} D_{t}^{\alpha_{l}} u^{k}\right|+\left|{ }_{0}^{C} D_{t}^{\alpha_{l}} u^{k}\right| \\
& \leq O\left(\tau^{2}\right)+\max _{0 \leq s \leq T}\left|{ }_{0}^{C} \mathcal{D}_{t}^{\alpha_{l}} u(s)\right| \\
& \leq 1+\max _{\substack{0 \leq s \leq T \\
0 \leq \alpha \leq 1}}\left|{ }_{0}^{C} \mathcal{D}_{t}^{\alpha} u(s)\right| .
\end{aligned}
$$

Invoking (4.3), 4.4) and (3.5), we find that

$$
\begin{aligned}
\left|\left(R_{2}^{k}, v\right)\right| & \leq c\left(1+\omega_{0}\right) h^{m+1}\left\|\frac{1}{\tau^{\alpha_{l}}} \sum_{j=0}^{k} \lambda_{j}^{\left(\alpha_{l}\right)} u^{k-j}\right\|_{m+1}\|v\| \\
& \leq c h^{m+1}\left(1+\max _{\substack{0 \leq s \leq T \\
0 \leq \alpha \leq 1}}\left\|\mathcal{D}_{t}^{\alpha} u(s)\right\|_{m+1}\right)\|v\| \\
& \leq c h^{2 m+2}\left(1+\max _{\substack{0 \leq s \leq T \\
0 \leq \alpha \leq 1}}\left\|_{0}^{C} \mathcal{D}_{t}^{\alpha} u(s)\right\|_{m+1}\right)^{2}+\frac{\beta_{1}}{8}\|\nabla v\|^{2}, \quad \forall 1 \leq k \leq N .
\end{aligned}
$$


The desired result follows on inserting (4.2) and 4.5 into 4.1. This completes the proof.

Our task now is to derive the supercloseness estimate of the scheme 3.6 .

Theorem 4.3. Suppose that ${ }_{0}^{C} D_{t}^{\alpha} u \in C^{2}\left([0, T] ; H^{m+1}(\Omega) \cap H_{0}^{1}(\Omega)\right), u_{t} \in H^{m+2}(\Omega) \cap$ $H_{0}^{1}(\Omega)$, let $U_{h}^{k}$ and $I_{h} u^{k}$ be the fully discrete finite element solution and finite element interpolation of $u^{k}$, respectively. Then we have the following supercloseness estimate for $1 \leq n \leq N$

$$
\begin{aligned}
& \tau \sum_{k=1}^{n}\left\|I_{h} u^{k}-U_{h}^{k}\right\|_{1} \\
\leq & c\left(\tau^{2}+(\Delta \alpha)^{2}+h^{m+1}\right)\left(1+\max _{\substack{0 \leq s \leq T \\
0 \leq \alpha \leq 1}}\left\|_{0}^{C} \mathcal{D}_{t}^{\alpha} u(s)\right\|_{m+1}+\int_{0}^{T}\left\|u_{t}(s)\right\|_{m+2} d s\right) .
\end{aligned}
$$

Proof. For convenience, we employ the following splitting of the error

$$
e^{k}=\left(u^{k}-I_{h} u^{k}\right)+\left(I_{h} u^{k}-U_{h}^{k}\right) \equiv \rho^{k}+\theta^{k} .
$$

Taking an inner product of (3.1) with $v_{h} \in V_{h}$ and comparing with (3.6), we obtain the error equation for $1 \leq k \leq N$

$$
\begin{aligned}
& \Delta \alpha \sum_{l=0}^{2 J} c_{l} w\left(\alpha_{l}\right) \frac{1}{\tau^{\alpha_{l}}} \sum_{j=0}^{k} \lambda_{j}^{\left(\alpha_{l}\right)}\left(\theta^{k-j}, v_{h}\right)+\left(b \nabla \theta^{k}, \nabla v_{h}\right) \\
= & -\left(R^{k}, v_{h}\right)-\left(b \nabla \rho^{k}, \nabla v_{h}\right), \quad \forall v_{h} \in V_{h},
\end{aligned}
$$

where

$$
R^{k}=\mathcal{D}_{t}^{[w]} u^{k}-\Delta \alpha \sum_{l=0}^{2 J} c_{l} w\left(\alpha_{l}\right) \frac{1}{\tau^{\alpha_{l}}} \sum_{j=0}^{k} \lambda_{j}^{\left(\alpha_{l}\right)} I_{h} u^{k-j} .
$$

Taking $v_{h}=\theta^{k}$ in 4.6 , then it follows that

$$
\begin{aligned}
& \Delta \alpha \sum_{l=0}^{2 J} c_{l} w\left(\alpha_{l}\right) \frac{1}{\tau^{\alpha_{l}}} \sum_{j=0}^{k} \lambda_{j}^{\left(\alpha_{l}\right)}\left(\theta^{k-j}, \theta^{k}\right)+\left(b \nabla \theta^{k}, \nabla \theta^{k}\right) \\
= & -\left(R^{k}, \theta^{k}\right)-\left(b \nabla \rho^{k}, \nabla \theta^{k}\right), \quad \forall 1 \leq k \leq N .
\end{aligned}
$$

Let $\bar{b}=\frac{1}{|K|} \int_{K} b(x, y) d x d y$. Applying the Bramble-Hilbert Lemma (cf. [5]), it holds that

$$
\|b(x, y)-\bar{b}\|_{0, \infty} \leq \operatorname{ch}\|b\|_{1, \infty}
$$


Using the Cauchy-Schwarz inequality, Lemma 4.1 and (4.8), we arrive at

$$
\begin{aligned}
\left|-\left(b \nabla \rho^{k}, \nabla \theta^{k}\right)\right| & =\left|\left((b-\bar{b}) \nabla \rho^{k}, \nabla \theta^{k}\right)+\left(\bar{b} \nabla \rho^{k}, \nabla \theta^{k}\right)\right| \\
& =\left|\left((b-\bar{b}) \nabla \rho^{k}, \nabla \theta^{k}\right)+\sum_{K \in \Im_{h}} \bar{b} \int_{K} \nabla \rho^{k} \cdot \nabla \theta^{k} d x d y\right| \\
& \leq c h\|b\|_{1, \infty}\left\|\nabla \rho^{k}\right\|\left\|\nabla \theta^{k}\right\|+c h^{m+1}\left\|u^{k}\right\|_{m+2}\left\|\nabla \theta^{k}\right\| \\
& \leq c h^{m+1}\left\|u^{k}\right\|_{m+2}\left\|\nabla \theta^{k}\right\| \\
& \leq c h^{2 m+2}\left\|u^{k}\right\|_{m+2}^{2}+\frac{\beta_{1}}{4}\left\|\nabla \theta^{k}\right\|^{2} \\
& \leq c h^{2 m+2}\left(\left\|u^{0}\right\|_{m+2}+\int_{0}^{t_{k}}\left\|u_{t}(s)\right\|_{m+2} d s\right)^{2}+\frac{\beta_{1}}{4}\left\|\nabla \theta^{k}\right\|^{2} .
\end{aligned}
$$

Substituting (4.9) and Lemma 4.2 into 4.7), and summing up $k$ from 1 to $n$, we obtain

$$
\begin{aligned}
& \Delta \alpha \sum_{l=0}^{2 J} c_{l} w\left(\alpha_{l}\right) \frac{1}{\tau^{\alpha_{l}}} \sum_{k=1}^{n} \sum_{j=0}^{k} \lambda_{j}^{\left(\alpha_{l}\right)}\left(\theta^{k-j}, \theta^{k}\right)+\beta_{1} \sum_{k=1}^{n}\left\|\nabla \theta^{k}\right\|^{2} \\
& \leq c\left(\tau^{2}+(\Delta \alpha)^{2}+h^{m+1}\right)^{2} \sum_{k=1}^{n}\left(1+\max _{\substack{0 \leq s \leq T \\
0 \leq \alpha \leq 1}}\left\|_{0}^{C} \mathcal{D}_{t}^{\alpha} u(s)\right\|_{m+1}+\int_{0}^{t_{k}}\left\|u_{t}(s)\right\|_{m+2} d s\right)^{2} \\
& +\frac{\beta_{1}}{2} \sum_{k=1}^{n}\left\|\nabla \theta^{k}\right\|^{2}, \quad \forall 1 \leq n \leq N .
\end{aligned}
$$

Adding $\mu\left(\theta^{0}, \theta^{0}\right)$ on both sides of the above inequality, we have

$$
\begin{aligned}
& \Delta \alpha \sum_{l=0}^{2 J} c_{l} w\left(\alpha_{l}\right) \frac{1}{\tau^{\alpha_{l}}} \sum_{k=0}^{n} \sum_{j=0}^{k} \lambda_{j}^{\left(\alpha_{l}\right)}\left(\theta^{k-j}, \theta^{k}\right)+\beta_{1} \sum_{k=1}^{n}\left\|\nabla \theta^{k}\right\|^{2} \\
& \leq c\left(\tau^{2}+(\Delta \alpha)^{2}+h^{m+1}\right)^{2} \sum_{k=1}^{n}\left(1+\max _{\substack{0 \leq s \leq T \\
0 \leq \alpha \leq 1}}\left\|_{0}^{C} \mathcal{D}_{t}^{\alpha} u(s)\right\|_{m+1}+\int_{0}^{t_{k}}\left\|u_{t}(s)\right\|_{m+2} d s\right)^{2} \\
& +\mu\left(\theta^{0}, \theta^{0}\right)+\frac{\beta_{1}}{2} \sum_{k=1}^{n}\left\|\nabla \theta^{k}\right\|^{2}, \quad \forall 1 \leq n \leq N .
\end{aligned}
$$

Applying Lemma 2.4 yields the desired result. This completes the proof.

Furthermore, we can construct the interpolation postprocessing operator $\Pi_{2 h}$ to obtain the following global superconvergent result of the fully discrete scheme as [16]. For completeness, we describe the construction method of the postprocessing interpolation operator $\Pi_{2 h}$. First we combine four neighboring elements into a big element $\widetilde{K}=\bigcup_{i=1}^{4} K_{i}$, 
which has the following properties (cf. [16,21])

$$
\begin{cases}\left.\Pi_{2 h} u\right|_{\widetilde{K}} \in Q_{m+1}(\widetilde{K}) & \forall \widetilde{K} \in \Im_{2 h}, \\ \Pi_{2 h} I_{h} u=\Pi_{2 h} u & \forall u \in C(\bar{\Omega}), \\ \left\|\Pi_{2 h} u-u\right\|_{1} \leq c h^{m+1}\|u\|_{m+2} & \forall u \in H^{m+2}(\Omega), \\ \left\|\Pi_{2 h} v_{h}\right\|_{1} \leq c\left\|v_{h}\right\|_{1} & \forall v_{h} \in V_{h},\end{cases}
$$

where $Q_{m+1}(\widetilde{K})$ is a space polynomials whose degrees for $x, y$ are no more than $m+1$, respectively, $\Im_{2 h}$ consists of the four small elements $K_{i}(i=1,2,3,4)$ in $\Im_{h}$.

Theorem 4.4. Under the conditions of Theorem 4.3, then we have the following superconvergence result for $1 \leq n \leq N$

$$
\begin{aligned}
& \tau \sum_{k=1}^{n}\left\|u^{k}-\Pi_{2 h} U_{h}^{k}\right\|_{1} \\
\leq & c\left(\tau^{2}+(\Delta \alpha)^{2}+h^{m+1}\right)\left(1+\max _{\substack{0 \leq s \leq T \\
0 \leq \alpha \leq 1}}\left\|_{0}^{C} \mathcal{D}_{t}^{\alpha} u(s)\right\|_{m+1}+\int_{0}^{T}\left\|u_{t}(s)\right\|_{m+2} d s\right) .
\end{aligned}
$$

Proof. We can deduce from the property 4.10 and Theorem 4.3 that

$$
\begin{aligned}
& \tau \sum_{k=1}^{n}\left\|u^{k}-\Pi_{2 h} U_{h}^{k}\right\|_{1} \\
\leq & \tau \sum_{k=1}^{n}\left\|u^{k}-\Pi_{2 h} I_{h} u^{k}\right\|_{1}+\tau \sum_{k=1}^{n}\left\|\Pi_{2 h} I_{h} u^{k}-\Pi_{2 h} U_{h}^{k}\right\|_{1} \\
\leq & \tau \sum_{k=1}^{n}\left\|u^{k}-\Pi_{2 h} u^{k}\right\|_{1}+\tau \sum_{k=1}^{n}\left\|I_{h} u^{k}-U_{h}^{k}\right\|_{1} \\
\leq & c\left(\tau^{2}+(\Delta \alpha)^{2}+h^{m+1}\right)\left(1+\underset{\substack{0 \leq s \leq T \\
0 \leq \alpha \leq 1}}{\max }\left\|_{0}^{C} \mathcal{D}_{t}^{\alpha} u(s)\right\|_{m+1}+\int_{0}^{T}\left\|u_{t}(s)\right\|_{m+2} d s\right) .
\end{aligned}
$$

This completes the proof.

\section{Numerical experiment}

In this section, test example based on piecewise bilinear polynomials will be performed to illustrate the computational efficiency and convergence rate of the proposed method.

Example 5.1. Let $\Omega=[0, \pi] \times[0, \pi], T=0.5, b(x, y)=\sin x \sin y+0.1, w(\alpha)=\Gamma(\beta+1-\alpha)$ and initial conditions $u_{0}(x, y)=0$ and $\psi(x, y)=0$ in the problem 3.1 , then the exact solution of the example is

$$
u(x, y, t)=(2 t)^{\beta} \sin x \sin y,
$$


where $\beta$ is a positive constant. Then $f(x, y, t)$ is chosen corresponding to the exact solution.

\begin{tabular}{ccc}
\hline$\tau$ & $\left\|u^{n}-U_{h}^{n}\right\|_{1}$ & rate \\
\hline $1 / 2$ & $2.8602 \mathrm{e}-2$ & 2.07 \\
$1 / 4$ & $6.8097 \mathrm{e}-3$ & 2.03 \\
$1 / 8$ & $1.6620 \mathrm{e}-3$ & 2.04 \\
$1 / 16$ & $4.0529 \mathrm{e}-4$ & 2.10 \\
$1 / 32$ & $9.4220 \mathrm{e}-5$ & $*$ \\
\hline
\end{tabular}

Table 5.1: Numerical errors and convergence orders in temporal direction with $h=\pi / 300$, $\Delta \alpha=1 / 200$ and $\beta=3$.

Our first concern is to test the numerical convergence rate of the proposed scheme in time. Taking the fixed and sufficiently small $h$ and $\Delta \alpha$, the numerical errors and convergence orders in $H^{1}$ norm are given in Table 5.1. The space step size $h$ and distributed-order step size $\Delta \alpha$ are fixed to be sufficiently small to ensure that the dominant numerical errors come from the approximation of time-fractional derivatives. From Table 5.1, the second-order accuracy of scheme 3.6 in time is verified by the example.

Secondly, the numerical accuracies of the fully finite element scheme in space is verified by the example. With fixed sufficiently small temporal stepsizes, the $\left\|u^{n}-U_{h}^{n}\right\|,\left\|u^{n}-U_{h}^{n}\right\|_{1}$, $\left\|I_{h} u^{n}-U_{h}^{n}\right\|_{1}$ and $\left\|u^{n}-\Pi_{2 h} U_{h}^{n}\right\|_{1}$ norm errors and spatial convergence orders of the scheme are illustrated in Table 5.2. As predicted by the theoretical estimates, the second-order supercloseness and superconvergence of the scheme in space variable for computing this example are verified.

\begin{tabular}{ccccccccc}
\hline$h$ & $\left\|u^{n}-U_{h}^{n}\right\|$ & rate & $\left\|u^{n}-U_{h}^{n}\right\|_{1}$ & rate & $\left\|I_{h} u^{n}-U_{h}^{n}\right\|_{1}$ & rate & $\left\|u^{n}-\Pi_{2 h} U_{h}^{n}\right\|_{1}$ & rate \\
\hline$\pi / 4$ & $2.7783 \mathrm{e}-1$ & 1.96 & $6.4095 \mathrm{e}-1$ & 0.99 & $8.8916 \mathrm{e}-2$ & 2.13 & $7.3428 \mathrm{e}-2$ & 1.95 \\
$\pi / 8$ & $7.1625 \mathrm{e}-2$ & 1.99 & $3.2382 \mathrm{e}-1$ & 1.00 & $2.0343 \mathrm{e}-2$ & 2.04 & $1.8993 \mathrm{e}-2$ & 1.96 \\
$\pi / 16$ & $1.8037 \mathrm{e}-2$ & 2.00 & $1.6239 \mathrm{e}-1$ & 1.00 & $4.9636 \mathrm{e}-3$ & 2.01 & $4.8653 \mathrm{e}-3$ & 1.99 \\
$\pi / 32$ & $4.5157 \mathrm{e}-3$ & 2.00 & $8.1256 \mathrm{e}-2$ & 1.00 & $1.2311 \mathrm{e}-3$ & 2.01 & $1.2246 \mathrm{e}-3$ & 2.01 \\
$\pi / 64$ & $1.1277 \mathrm{e}-3$ & $*$ & $4.0636 \mathrm{e}-2$ & $*$ & $3.0493 \mathrm{e}-4$ & $*$ & $3.0451 \mathrm{e}-4$ & $*$ \\
\hline
\end{tabular}

Table 5.2: Numerical errors and convergence orders in spatial direction with $\tau=1 / 200$, $\Delta \alpha=1 / 200$ and $\beta=3$.

Thirdly, we would like to investigate the numerical accuracy of the scheme 3.6 in distributed-order variable. The $H^{1}$ norm errors decrease as the step sizes in distribute order are reduced. The second-order numerical convergence rate of the scheme (3.6) in 
distributed order can be well visible from Table 5.3 .

\begin{tabular}{ccc}
\hline$\Delta \alpha$ & $\left\|u^{n}-U_{h}^{n}\right\|_{1}$ & rate \\
\hline $1 / 2$ & $1.4746 \mathrm{e}-2$ & 2.00 \\
$1 / 4$ & $3.6936 \mathrm{e}-3$ & 2.08 \\
$1 / 8$ & $8.7375 \mathrm{e}-4$ & 2.10 \\
$1 / 16$ & $2.0423 \mathrm{e}-4$ & 1.99 \\
$1 / 32$ & $5.1254 \mathrm{e}-5$ & $*$ \\
\hline
\end{tabular}

Table 5.3: Numerical errors and convergence orders in distributed order with $h=\pi / 300$, $\tau=1 / 200$ and $\beta=3$.

\begin{tabular}{c|ccc}
\hline$\beta$ & $\tau$ & $\left\|u^{n}-U_{h}^{n}\right\|_{1}$ & rate \\
\hline \multirow{5}{*}{$\beta=2$} & $1 / 2$ & $2.6046 \mathrm{e}-2$ & 1.81 \\
& $1 / 4$ & $7.4269 \mathrm{e}-3$ & 1.86 \\
& $1 / 8$ & $2.0430 \mathrm{e}-3$ & 1.88 \\
& $1 / 16$ & $5.5631 \mathrm{e}-4$ & 1.91 \\
& $1 / 32$ & $1.4851 \mathrm{e}-4$ & $*$ \\
\hline \multirow{5}{*}{$\beta=3 / 2$} & $1 / 2$ & $1.0850 \mathrm{e}-2$ & 1.50 \\
& $1 / 4$ & $3.8245 \mathrm{e}-3$ & 1.16 \\
& $1 / 16$ & $1.7168 \mathrm{e}-3$ & 1.18 \\
& $1 / 32$ & $3.2251 \mathrm{e}-4$ & $*$ \\
\hline \multirow{5}{*}{$\beta=1$} & $1 / 2$ & $1.8643 \mathrm{e}-1$ & 0.78 \\
& $1 / 4$ & $1.0873 \mathrm{e}-1$ & 0.81 \\
& $1 / 8$ & $6.1966 \mathrm{e}-2$ & 0.86 \\
& $1 / 16$ & $3.4045 \mathrm{e}-2$ & 0.90 \\
& $1 / 32$ & $1.8235 \mathrm{e}-3$ & $*$ \\
\hline
\end{tabular}

Table 5.4: Numerical errors and convergence orders in temporal direction under $h=$ $\pi / 300, \Delta \alpha=1 / 200$ with different constants $\beta$.

To this end, in this example, we are concerned with the computational results of the scheme (3.6) for the cases $\beta=2,3 / 2,1$, respectively. Table 5.4 lists the computational errors and convergence orders in time variable for these three cases, from which, one can 
find that the second-order convergence of the scheme in time variable can still be achieved for the case $\beta=2$, whereas the numerical accuracy is obviously reduced to less than

two for the latter two cases. We have pointed out that the condition $\left.\frac{\partial^{n} u(x, y, t)}{\partial t^{n}}\right|_{t=0}=0$ $(n=0,1, \ldots, 4)$ is sufficient but unnecessary for the numerical accuracy of the proposed scheme.

\section{Conclusions}

In this paper, we have dealt with the numerical solutions to some distributed order time fractional diffusion equations. An effective Galerkin finite element fully discrete scheme is developed for equations variable coefficient. The unconditional stability and superconvergence error estimates of the obtained scheme is investigated using integral identities and postprocessing techniques. The global second-order convergence in time variable of the obtained schemes is attainable. In Section 5, we have pointed out that the condition $\left.\frac{\partial^{n} u(x, y, t)}{\partial t^{n}}\right|_{t=0}=0(n=0,1, \ldots, 4)$ is sufficient but unnecessary for the numerical accuracy of the proposed scheme. Hence, the direct application of the shifted and weighted Grünwald Letnikov formula can not produce the ideal computational results to approximate the time fractional derivatives in (3.1). However, Alikhanov 2 derived a new numerical differentiation formula, called the $L 2-1_{\sigma}$ formula, to approximate the Caputo fractional derivative at a special point. And the time second-order difference scheme for fractional diffusion equations is developed and analyzed in [2]. It is possible that the $L 2-1_{\sigma}$ formula can solve nonzero boundary value problems. In future work, the numerical solutions for nonzero boundary value problems and the nonlinear fractional differential equations of distributed order will be investigated.

\section{Acknowledgments}

We would like to thank the anonymous referees for many constructive comments and suggestions which led to an improved presentation of this paper.

\section{References}

[1] R. A. Adams, Sobolev Spaces, Pure and Applied Mathematics 65, Academic Press, New York, 1975.

[2] A. A. Alikhanov, A new difference scheme for the time fractional diffusion equation, J. Comput. Phys. 280 (2015), 424-438.

[3] A. V. Chechkin, R. Gorenflo and I. M. Sokolov, Retarding subdiffusion and accelerating superdiffusion governed by distributed-order fractional diffusion equations, Phys. Rev. E 66 (2002), no. 4, 046129. 
[4] A. V. Chechkin, R. Gorenflo, I. M. Sokolov and V. Y. Gonchar, Distributed order time fractional diffusion equation, Fract. Calc. Appl. Anal. 6 (2003), no. 3, 259-279.

[5] P. G. Ciarlet and J. L. Lions, Handbook of Numerical Analysis II: Finite element methods, Amsterdam, North-Holland, 1991.

[6] M. Cui, Compact alternating direction implicit method for two-dimensional time fractional diffusion equation, J. Comput. Phys. 231 (2012), no. 6, 2621-2633.

[7] K. Diethelm and N. J. Ford, Numerical analysis for distributed-order differential equations, J. Comput. Appl. Math. 225 (2009), no. 1, 96-104.

[8] H. Eichel, L. Tobiska and H. Xie, Supercloseness and superconvergence of stabilized low-order finite element discretizations of the Stokes problem, Math. Comp. 80 (2011), no. $274,697-722$.

[9] Q. Feng and F. Meng, Traveling wave solutions for fractional partial differential equations arising in mathematical physics by an improved fractional Jacobi elliptic equation method, Math. Methods Appl. Sci. 40 (2017), no. 10, 3676-3686.

[10] G.-H. Gao and Z.-Z. Sun, Two alternating direction implicit difference schemes for two-dimensional distributed-order fractional diffusion equations, J. Sci. Comput. 66 (2016), no. 3, 1281-1312.

[11] G.-H. Gao, H.-W. Sun and Z.-Z. Sun, Some high-order difference schemes for the distributed-order differential equations, J. Comput. Phys. 298 (2015), 337-359.

[12] L. Guo, L. Liu and Y. Wu, Existence of positive solutions for singular fractional differential equations with infinite-point boundary conditions, Nonlinear Anal. Model. Control. 21 (2016), no. 5, 635-650.

[13] M. Li and J. Wang, Exploring delayed Mittag-Leffler type matrix functions to study finite time stability of fractional delay differential equations, Appl. Math. Comput. 324 (2018), 254-265.

[14] H.-L. Liao, P. Lyu, S. Vong and Y. Zhao, Stability of fully discrete schemes with interpolation-type fractional formulas for distributed-order subdiffusion equations, $\mathrm{Nu-}$ mer. Algorithms 75 (2017), no. 4, 845-878.

[15] H.-L. Liao, Y.-N. Zhang, Y. Zhao and H.-S. Shi, Stability and convergence of modified Du Fort-Frankel schemes for solving time-fractioal subdiffusion equations, J. Sci. Comput. 61 (2014), no. 3, 629-648. 
[16] Q. Lin and N. N. Yan, The Construction and Analysis of High Efficient Elements, Hebei University Press, China, 1996.

[17] F. Liu, M. M. Meerschaert, R. J. McGough, P. Zhuang and Q. Liu, Numerical methods for solving the multi-term time-fractional wave-diffusion equation, Fract. Calc. Appl. Anal. 16 (2013), no. 1, 9-25.

[18] C. Lubich, Discretized fractional calculus, SIAM J. Math. Anal. 17 (1986), no. 3, 704-719.

[19] M. M. Meerschaert and C. Tadjeran, Finite difference approximations for fractional advection-dispersion flow equations, J. Comput. Appl. Math. 172 (2004), no. 1, 65-77.

[20] I. Podlubny, Fractional Differential Equations: An introduction to fractional derivatives, fractional differential equations, to methods of their solution and some of their applications, Mathematics in Science and Engineering 198, Academic Press, San Diego, CA, 1999.

[21] J. Ren and Y. Ma, A superconvergent nonconforming mixed finite element method for the Navier-Stokes equations, Numer. Methods Partial Differential Equations 32 (2016), no. 2, 646-660.

[22] J. Ren and Z.-Z. Sun, Efficient and stable numerical methods for multi-term time fractional sub-diffusion equations, East Asian J. Appl. Math. 4 (2014), no. 3, 242266.

[23]__fficient numerical solution of the multi-term time fractional diffusion-wave equation, East Asian J. Appl. Math. 5 (2015), no. 1, 1-28.

[24] S. G. Samko, A. A. Kilbas and O. I. Marichev, Fractional Integrals and Derivatives: Theory and applications, Gordon and Breach, London, 1993.

[25] T. Shen, J. Xin and J. Huang, Time-space fractional stochastic Ginzburg-Landau equation driven by Gaussian white noise, Stoch. Anal. Appl. 36 (2018), no. 1, 103113.

[26] W. Tian, H. Zhou and W. Deng, A class of second order difference approximations for solving space fractional diffusion equations, Math. Comp. 84 (2015), no. 294, 1703-1727.

[27] S. Vong and Z. Wang, A compact difference scheme for a two dimensional fractional Klein-Gordon equation with Neumann boundary conditions, J. Comput. Phys. 274 (2014), 268-282. 
[28] Z. Wang and S. Vong, Compact difference schemes for the modified anomalous fractional sub-diffusion equation and the fractional diffusion-wave equation, J. Comput. Phys. 277 (2014), 1-15.

[29] H. Ye, F. Liu, V. Anh and I. Turner, Numerical analysis for the time distributed-order and Riesz space fractional diffusions on bounded domains, IMA J. Appl. Math. 80 (2015), no. 3, 825-838.

[30] X. Zhang, L. Liu and Y. Wu, Multiple positive solutions of a singular fractional differential equation with negatively perturbed term, Math. Comput. Modelling $\mathbf{5 5}$ (2012), no. 3-4, 1263-1274.

[31] _ Existence results for multiple positive solutions of nonlinear higher order perturbed fractional differential equations with derivatives, Appl. Math. Comput. 219 (2012), no. 4, 1420-1433.

[32] J. Zhang, Z. Lou, Y. Ji and W. Shao, Ground state of Kirchhoff type fractional Schrödinger equations with critical growth, J. Math. Anal. Appl. 462 (2018), no. 1, $57-83$.

[33] Y.-N. Zhang and Z.-Z. Sun, Alternating direction implicit schemes for the twodimensional fractional sub-diffusion equation, J. Comput. Phys. 230 (2011), no. 24, 8713-8728.

[34] Y.-N. Zhang, Z.-Z. Sun and H.-W. Wu, Error estimates of Crank-Nicolson-type difference schemes for the subdiffusion equation, SIAM J. Numer. Anal. 49 (2011), no. 6, 2302-2322.

[35] P. Zhuang, F. Liu, V. Anh and I. Turner, New solution and analytical techniques of the implicit numerical method for the anomalous subdiffusion equation, SIAM J. Numer. Anal. 46 (2008), no. 2, 1079-1095.

Yanhua Yang

Department Information Engineering, Xinyang Agriculture and Forestry University,

Xinyang 464000, China

E-mail address: hl465331@163.com

Jincheng Ren

College of Mathematics and Information Science, Henan University of Economics and

Law, Zhengzhou 450045, China

E-mail address: renjincheng2001@126.com 